\title{
Simulación del Proceso de Absorción Química con Soluciones de Aminas para la Purificación Biogás
}

\author{
Betzabet Morero $^{(1)}$ y Enrique A. Campanella ${ }^{(1,2)}$ \\ (1) Universidad Nacional del Litoral-CONICET, Instituto de Desarrollo Tecnológico para la Industria Química \\ (INTEC), Güemes 3450, (3000) Santa Fe-Argentina. (e-mail: bmorero@intec.unl.edu.ar). \\ (2) Universidad Nacional del Litoral, Facultad de Ingeniería y Ciencias Hídricas, Ruta Nacional № $168 \mathrm{Km}$ \\ 472.4, (3000) Santa Fe-Argentina. (e-mail: tquique@santafe-conicet.gov.ar).
}

Recibido Jun. 22, 2012; Aceptado Jul. 24, 2012; Versión final recibida Ago. 17, 2012

\begin{abstract}
Resumen
Se analiza la influencia de los principales parámetros de operación y de distintos tipos de aminas en la purificación de biogás. Para ello se simuló un proceso convencional de absorción-desorción usando cuatro tipos de aminas (monoetanolamina, dietanolamina, diglicolamina y metildietanolamina) y dos mezclas de ellas. Para los cálculos se usó el simulador de procesos ProMax®. Las variables analizadas fueron la concentración de metano, anhídrido carbónico, ácido sulfhídrico y agua en el gas purificado, el consumo de energía en el proceso, la temperatura de absorción y la tasa de vapor utilizada en el vaporizador. En todos los casos se obtuvieron buenos resultados en los niveles de purificación alcanzados, los mayores niveles se obtuvieron con diglicolamina $\left(97.3 \%\right.$ de $\left.\mathrm{CH}_{4}\right)$ que además presenta el menor gasto energético en el vaporizador. Los conocimientos previos sobre sistemas de absorción-desorción de gas natural permitieron adaptar la tecnología al caso particular del biogás.
\end{abstract}

Palabras clave: simulación de procesos, Promax, purificación de biogás, soluciones de aminas

\section{Simulation of the Process of Chemical Absorption using Amine Solutions for Biogas Purification}

\begin{abstract}
The influence of some key operating parameters and of different types of amines in biogas purification is analyzed. For this a conventional single-loop absorber-stripper process configuration was simulated using four types of amines (monoethanolamine, diethanolamine, diglycolamine and methyldiethanolamine) and two mixtures of them. The process simulator ProMax ${ }^{\circledR}$ was used. The variables studied were methane, carbon dioxide, hydrogen sulphide and water concentrations in the sweetened gas, process energy consumption, absorption temperatures and reboiler steam rates. The simulation results showed good levels of purification in all cases with best results for diglycolamine $\left(97.3 \% \mathrm{de}^{\mathrm{C}} \mathrm{H}_{4}\right)$ which also has the lowest reboiler energy consumption. Prior knowledge of natural gas absorber-stripper processes allowed using the technology in the particular case of biogas.
\end{abstract}

Keywords: process simulation, Promax, biogas purification, amine solutions 


\section{INTRODUCCIÓN}

La alta dependencia que existe actualmente de los combustibles fósiles como fuente primaria para la generación de energía determina la insustentabilidad de la matriz energética. Por lo tanto, la sustitución de los hidrocarburos por fuentes alternativas sustentables de generación de energía se ha transformado en una prioridad de los últimos años. El biogás, que se produce a partir de la digestión anaeróbica de residuos orgánicos, es una opción muy interesante ya que puede provenir de diferentes fuentes (industriales, municipales y/o agrícolas), resolviendo además los problemas relacionados con su contaminación. Por otra parte, contribuye a disminuir el calentamiento global al reducir la alta dependencia con los hidrocarburos y al aprovechar el metano eliminado por dichos desperdicios.

La composición aproximada del biogás se estima en $60 \%$ de metano $\left(\mathrm{CH}_{4}\right), 35 \%$ de anhídrido carbónico $\left(\mathrm{CO}_{2}\right), 4 \%$ de vapor de agua y un máximo estimado de $1 \%$ de ácido sulfhídrico $\left(\mathrm{H}_{2} \mathrm{~S}\right)$. Estos compuestos diferentes al metano pueden resultar perjudiciales para los equipos que finalmente utilizan biogás, causando problemas de corrosión, o simplemente disminuyendo la eficiencia del equipo, ya que el metano es el único compuesto con propiedades combustibles. Además, dependiendo de la procedencia del biogás, puede contener ciertos compuestos que pueden resultar nocivos para la salud de las personas. Por esto, cuanto mayor sea la concentración de metano en el biogás, mejor será su aprovechamiento como fuente alternativa de energía segura y sustentable.

Entre los sistemas de purificación existentes actualmente se encuentra, por ejemplo, el proceso Binax, desarrollado por Central Plants Inc. en California, USA (Henrich, 1983). Este esquema puede perfeccionarse con un rediseño de la torre de absorción (Rasi et al., 2008). Por otro lado, tanto la empresa Guild Associates, Inc. (Ohio, USA) como la HAASE Energietechnik AG (Alemania) ofrecen un proceso de purificación en el cual, se comprime biogás a alta presión, como primera etapa, y luego se introduce a un sistema de adsorción. Por otro parte, Abatzoglou (2009), Patterson el al. (2011) y Ryckebosch et al. (2011) discuten diferentes métodos para la purificación y la mejora de las técnicas existentes. Recientemente, Varnero et.al. (2012) revisa diferentes tecnologías de purificación de biogás para su posterior uso como generador de energía eléctrica. Además Tippayawong y Thanompongchart (2010) han investigado la eliminación del $\mathrm{CO}_{2}$ y el $\mathrm{H}_{2} \mathrm{~S}$ con soluciones acuosas de sales y aminas en una columna empacada, aunque recomiendan que se continúe investigando la factibilidad y eficiencia de esta tecnología a grandes escalas.

Las aminas han sido ampliamente estudiadas por muchos años para remover las impurezas del gas natural (Kohl y Nielsen, 1997), principalmente $\mathrm{CO}_{2}$ y $\mathrm{H}_{2} \mathrm{~S}$, demostrando buenos resultados para tal fin. También se han utilizado en los últimos años en la captura de $\mathrm{CO}_{2}$ de los gases de combustión eliminados en las plantas de generación de energía a partir de combustibles fósiles (Abu-Zahra, et al., 2007; Nuchitprasittichai y Cremaschi, 2011). La captura de $\mathrm{CO}_{2}$ es cada vez de mayor interés ya que se la considera un método potencial para el control de las emisiones de gases de efecto invernadero (Rubin et. al., 2012). Si bien esta tecnología madura ya se aplica en muchas plantas industriales, aún no se ha profundizado su estudio para el caso particular del biogás. La diferencia principal es que el biogás contiene concentraciones de $\mathrm{CO}_{2}$ cercanas al $40 \%$ mientras que las concentraciones en el gas natural y en los gases de combustión son inferiores al $15 \%$.

El objetivo de este trabajo es comparar la eficiencia de distintos tipos de aminas y discutir la influencia de los principales parámetros operativos en la purificación de biogás. Las variables analizadas para comparar las aminas fueron: la concentración de metano, anhídrido carbónico, ácido sulfhídrico y agua en el gas endulzado. Además se analizó el consumo de energía en el proceso, la temperatura de absorción y la tasa de vapor utilizada en el vaporizador. El simulador que se utilizó fue ProMax® con TSWEET® y PROSIM® ya que ha demostrado ser confiable en el diseño de unidades de endulzamiento de gas natural con aminas y las capacidades de ProMax han sido descritas previamente por varios autores (Holmes et al., 1984; Polasek et al., 1982, 1983). El simulador fue calibrado previamente con datos de la literatura. Los resultados obtenidos de la simulación demostraron que las aminas son una buena opción para la purificación del biogás ya que se alcanzaron altos niveles de remoción de impurezas.

\section{METODOLOGÍA}

Como se mencionó anteriormente las aminas han sido utilizadas por muchos años como una buena opción para la remoción de impurezas del gas natural $\left(\mathrm{CO}_{2}\right.$ y $\left.\mathrm{H}_{2} \mathrm{~S}\right)$. Por esto, se analiza la eficiencia de seis tipos de aminas o mezclas de ellas (Monoetanolamina (MEA), Dietanolamina (DEA), Diglicolamina (DGA), Metildietanolamina (MDEA), MDEA+MEA, MDEA+DEA) en el proceso de purificación de biogás. Para comparar la factibilidad de las aminas en la purificación se simuló una unidad convencional de absorcióndesorción, utilizando ProMax ${ }^{\circledR}$ con TSWEET ${ }^{\circledR}$ y PROSIM ${ }^{\circledR}$. El esquema de procesos de la unidad de endulzamiento modelada con el simulador se muestra en la Fig.1. La presión de vapor de $\mathrm{H}_{2} \mathrm{~S}$ y $\mathrm{CO}_{2}$ sobre 
las soluciones de aminas es calculada utilizando el modelo electrolítico ELR (Extended Long Range). Este modelo calcula los coeficientes de actividad de la fase líquida para predecir el equilibrio de fases multicomponente y está basado en el modelo de Pitzer-Debye-Hückel. Un modelo cinético en el absorbedor (TSWEET Kinetics) predice los efectos del tiempo de residencia, la temperatura, la concentración de la solución, la presión y el tipo de amina en la velocidad de absorción del $\mathrm{CO}_{2}$.

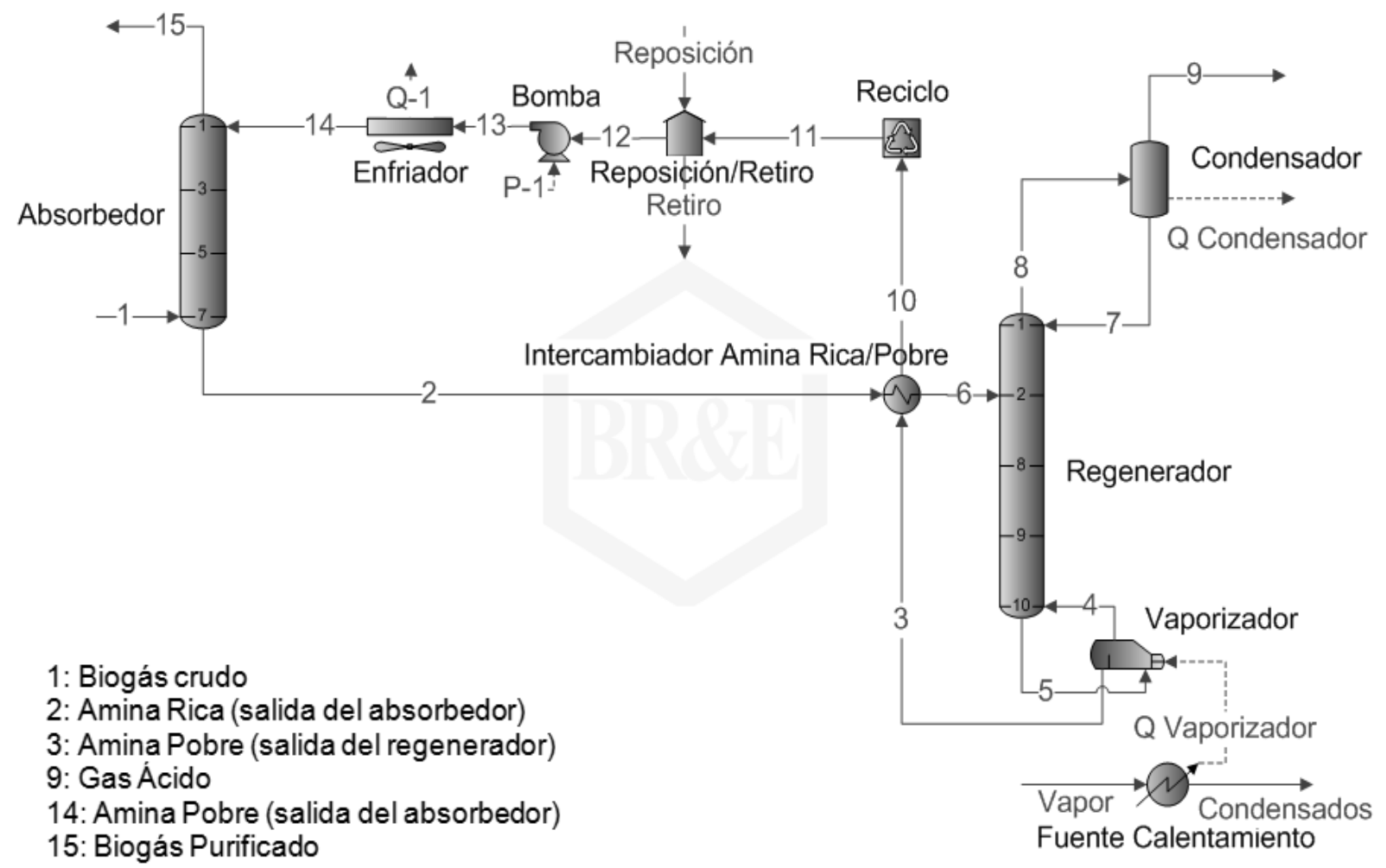

Fig. 1: Esquema del proceso convencional de absorción-desorción para la purificación de biogás.

Para simular correctamente la unidad de purificación, fue necesario estudiar las características de las aminas y su comportamiento en el proceso. Dicha información fue obtenida de la literatura (Polasek y Bullin, 1984; Kohl y Nielsen, 1997). Además se analizaron ciertos parámetros de operación propios del proceso. Los mismos se discutirán a continuación:

\section{Temperatura de absorción}

Este es un parámetro muy importante ya que controla la competencia entre el equilibrio termodinámico y la absorción limitada cinéticamente. Para el caso particular del $\mathrm{CO}_{2}$, un aumento en la temperatura generalmente aumenta en mayor medida los efectos cinéticos en relación con la disminución de la solubilidad. Sin embargo, luego de una cierta temperatura la solubilidad domina los efectos cinéticos. La modificación de este parámetro influye no solo en la cantidad de $\mathrm{CO}_{2}$ y $\mathrm{H}_{2} \mathrm{~S}$ absorbido sino también en las pérdidas de agua y aminas ya que estas aumentarán a medida que aumente la temperatura de absorción. Para el diseño y operación del equipo de absorción, se ha aconsejado por muchos años mantener una temperatura de aproximación de $10^{\circ} \mathrm{F}\left(5^{\circ} \mathrm{C}\right)$. Esta temperatura de aproximación se define como la diferencia de temperatura entre la corriente de gas ácido y la corriente de amina pobre. Esta regla de $5{ }^{\circ} \mathrm{C}$ se utiliza para evitar la condensación de hidrocarburos superiores en el absorbedor con las consiguientes pérdidas que esto produce, más la contaminación de la solución de aminas con hidrocarburos. El biogás contiene solamente metano el cual no es condensable a las condiciones de operación, por lo que no fue necesario respetar esta regla de oro de los procesos tradicionales de gas natural. Además, es bien conocido que en muchos casos disminuir la temperatura del absorbedor puede aumentar su eficiencia, especialmente para el caso de las aminas primarias y secundarias que cuentan con una pequeña participación cinética. Incluso para la MDEA, es posible aumentar el rendimiento en la absorción de $\mathrm{H}_{2} \mathrm{~S}$, cuya cinética es bastante rápida, a expensas del $\mathrm{CO}_{2}$ cuya absorción puede ser menor debido a los efectos de la temperatura en la velocidad cinética. 


\section{Tasa de vapor}

Es muy común encontrar en la literatura la tasa de vapor necesaria en las operaciones convencionales de regeneración, expresada en libras de vapor por galón de solución circulante. Los valores típicos varían entre 1 y 1,5 lb/gal aunque en ciertos casos puede llegar a 2 lb/gal. Si bien la carga de gas ácido en la amina pobre disminuye a medida que se aumenta la tasa de vapor al vaporizador, este valor está bastante atado a la economía del proceso ya que cuanto menor sea la tasa de vapor utilizada, menor será el requerimiento energético del vaporizador. Por lo que resulta conveniente no utilizar valores tan altos del mismo. Además, en la mayoría de los casos una tasa de vapor de aproximadamente 1,2 lb/gal es suficiente para obtener la pureza requerida del gas purificado, por lo que muchas plantas utilizan este valor (Kohl y Nielsen, 1997).

\section{Caudal de reciclo}

Para calcular el caudal de reciclo es necesario que el mismo cumpla con la condición de aproximación al método de equilibrio. Esta aproximación se basa en la premisa de que la máxima carga de la solución rica está dada por el equilibrio con el gas de alimentación en las condiciones del fondo del absorbedor. Dado que este máximo teórico no puede ser alcanzado en el equipo en la práctica, se asume una aproximación del $70-80 \%$. Esta aproximación puede ser considerada tanto en términos de la solución rica con una presión de vapor del gas ácido variando entre el 70-80\% de la presión parcial del mismo en la alimentación, o bien, en términos de la solución rica con una carga de gas ácido entre el $70-80 \%$ de la carga en el equilibrio con el gas de alimentación (Kohl y Nielsen, 1997). Por lo tanto, el caudal de reciclo (que es realmente la tasa de recirculación de la amina en el sistema) al respetar la aproximación al método de equilibrio garantiza que el sistema tenga la capacidad de responder ante incrementos de la concentración de gas ácido en la alimentación, sin disminuir la cantidad de metano obtenido en el gas purificado.

A partir de la información de los parámetros de operación discutidos se realizó la simulación utilizando la máxima concentración de aminas posible para evitar la corrosión. La composición de biogás utilizada se aproximó en: $60 \% \mathrm{CH}_{4}, 35 \% \mathrm{CO}_{2}, 4 \%$ vapor de agua, $1 \% \mathrm{H}_{2} \mathrm{~S}$. La corriente de ingreso de biogás crudo se fijó en $0,5 \mathrm{~m}^{3} / \mathrm{s}$ a $212^{\circ} \mathrm{F}$ de temperatura y 3 bares de presión (Shethna, H. y Towler, G., 1997), para todas las simulaciones. La temperatura de la corriente de amina al ingreso de la torre de absorción varió entre 110-150 ${ }^{\circ} \mathrm{F}$. El tiempo de residencia de cada plato teórico se fijó en 2,5 segundos. El caudal de reciclo se estableció en la simulación de manera tal que se operara a una condición de 70-80\% de la carga máxima de gas ácido de la amina rica y la tasa de vapor en el vaporizador se limitó en 1,2 lb/gal. En la Tabla 1 se resumen las características principales de la unidad de purificación para las diferentes aminas.

Tabla 1: Características principales de la unidad de absorción-desorción para las diferentes aminas a $110^{\circ} \mathrm{F}$.

\begin{tabular}{|l|cccccc|}
\hline Características & MEA & DEA & DGA & MDEA & MDEA+MEA & MDEA+DEA \\
\hline Concentración (\%) & 20 & 35 & 50 & 50 & $35+15$ & $35+15$ \\
Caudal de reciclo (gpm) & 120 & 100 & 80 & 90 & 90 & 110 \\
Carga máxima de gas ácido (\%) & 70,8 & 75 & 76,8 & 69 & 73,3 & 69 \\
$\begin{array}{l}\text { Carga amina rica (mol gas } \\
\text { ácido/mol amina) }\end{array}$ & 0,442 & 0,360 & 0,346 & 0,203 & 0,309 & 0,244 \\
\hline
\end{tabular}

En esta Tabla se observa que en la mayoría de los casos analizados se respeta la carga máxima recomendada para evitar la corrosión (0,35 moles de gas ácido/moles de amina), excepto en el caso de la MEA y DEA donde la carga de la corriente amina rica excede dicho valor, aunque este exceso es mínimo en el caso de la DEA.

\section{RESULTADOS Y DISCUSIÓN}

Lo primero que se consideró con los datos obtenidos de la simulación fue el comportamiento de las distintas aminas con la temperatura. En la Fig. 2 se puede observar la concentración de $\mathrm{CO}_{2}$ en el gas purificado donde se ve claramente el comportamiento diferente que presenta la MDEA frente a las otras tres aminas (MEA, DEA, DGA). Las aminas terciarias, y en especial la MDEA, tienen una baja velocidad de reacción (y por lo tanto de absorción) con $\mathrm{CO}_{2}$. Esto se debe a que la velocidad de reacción del $\mathrm{CO}_{2}$ disminuye con la sustitución del grupo nitrógeno por lo que las aminas primarias (MEA, DGA) reaccionan más rápido que las aminas secundarias (DEA) y a su vez estas lo hacen más rápido que las aminas terciarias (MDEA). En la Fig. 3 se puede apreciar cómo la adición de una amina primaria (MEA) o secundaria (DEA) a la MDEA aumenta la velocidad de absorción del $\mathrm{CO}_{2}$. 


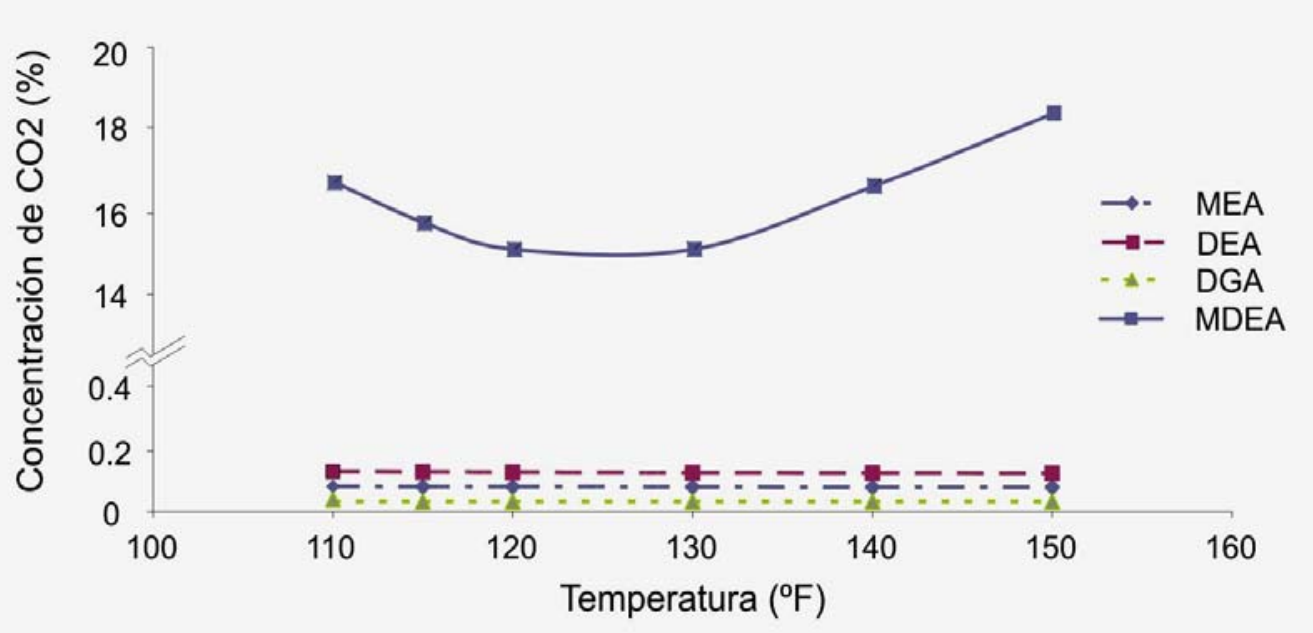

Fig. 2: Variación de la cantidad de $\mathrm{CO}_{2}$ absorbido a diferentes temperaturas por cuatro tipos de aminas.

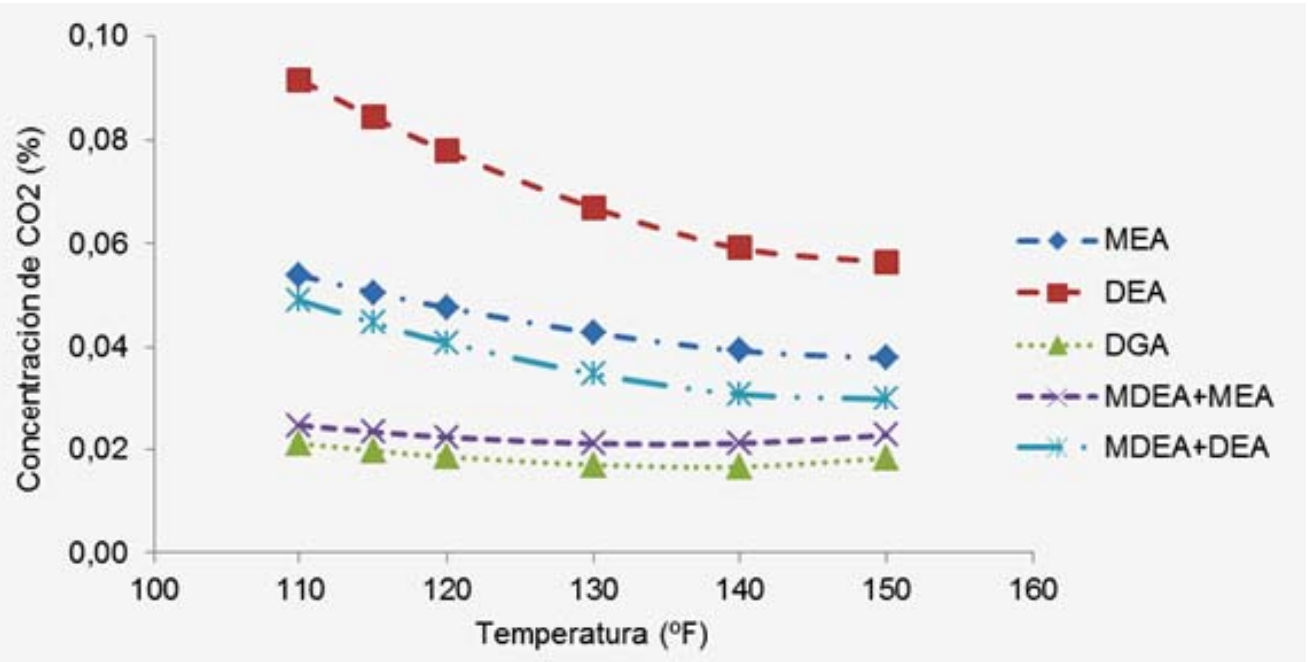

Fig. 3: Variación de la cantidad de $\mathrm{CO}_{2}$ absorbido a distintas temperaturas por diferentes tipos de aminas o mezclas de aminas.

Sin embargo, la disminución en la concentración de $\mathrm{CO}_{2}$ no es suficiente para alcanzar mayores niveles de purificación. En la Fig. 4 se observa cómo a medida que aumenta la temperatura, disminuye la concentración de metano en el biogás purificado (corriente 15). Esto se debe a que a medida que aumenta la temperatura en el absorbedor también aumenta la pérdida de agua, es decir, mayor es la concentración de agua en la corriente 15, tal como se grafica en la Fig. 5. Estas variaciones con respecto a la temperatura son muy similares a las que se producen en el gas natural (Lunsford y Bullin, 1996).

Estos resultados muestran que las aminas más eficientes para remover las impurezas son MEA, DEA y DGA siendo la MDEA la menos eficiente por lo que esta última amina debe mezclarse con alguna de las anteriores para obtener mejores resultados. Usar mezclas de aminas parece ser una buena opción cuando se desea remover en simultáneo $\mathrm{CO}_{2}$ y $\mathrm{H}_{2} \mathrm{~S}$, aunque los mayores niveles de metano se lograron con la DGA y con la mezcla MDEA+MEA. En la Tabla 2 se sintetiza la concentración final de la corriente 15 para las diferentes aminas o mezclas de ellas con las características mostradas en la Tabla 1.

El calor utilizado por el vaporizador es otro parámetro que se tuvo en cuenta para comparar los diferentes sistemas de endulzamiento con aminas. Los datos obtenidos mostraron que los procesos que consumen la menor cantidad de calor son lo que utilizan DGA (5,25 MMBTU/h) y la mezcla de aminas MDEA+MEA (5,91 MMBTU/h), mientras que el proceso que utiliza MEA es el que mayor cantidad de calor consume $(7,88$ MMBTU/h). 


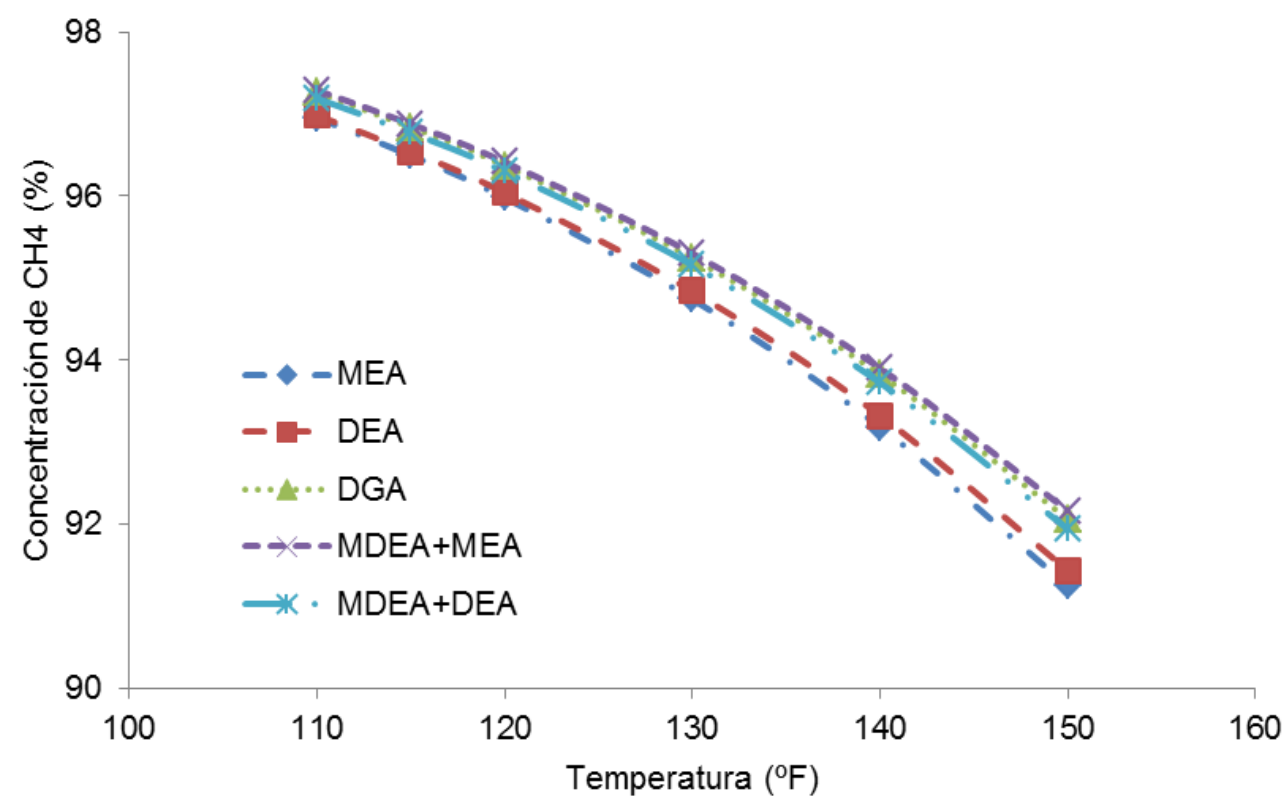

Fig. 4: Concentración de $\mathrm{CH}_{4}$ en el biogás purificado a diferentes temperaturas por distintos tipos de aminas o mezclas de aminas.

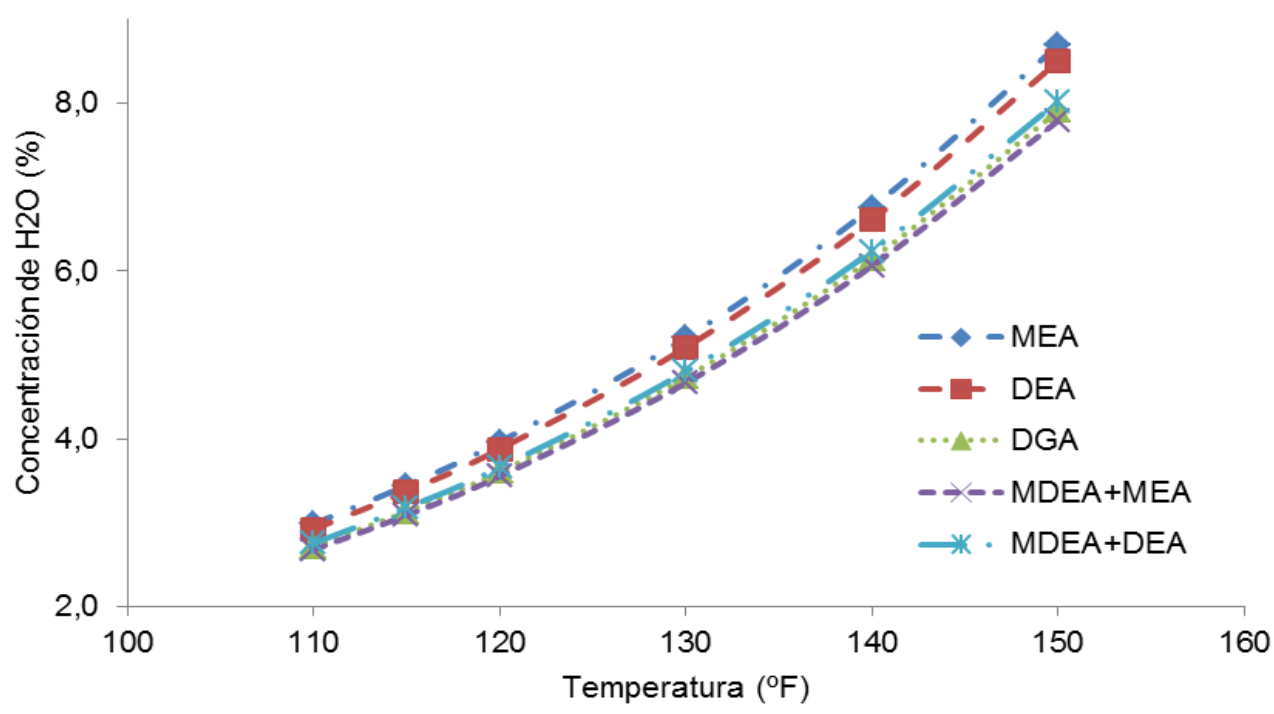

Fig. 5: Concentración de $\mathrm{H}_{2} \mathrm{O}$ en el biogás purificado a diferentes temperaturas por distintos tipos de aminas o mezclas de aminas.

Tabla 2: Concentración final del biogás purificado con diferentes aminas o mezclas de ellas.

\begin{tabular}{|l|cccc|}
\hline Tipo de Amina & $\mathrm{CH}_{4}(\%)$ & $\mathrm{CO}_{2}(\%)$ & $\mathrm{H}_{2} \mathrm{~S}(\mathrm{ppm})$ & $\mathrm{H}_{2} \mathrm{O}(\%)$ \\
\hline MEA & 97,0 & 0,05 & 0,28 & 2,99 \\
DEA & 97,0 & 0,09 & 0,70 & 2,93 \\
DGA & 97,3 & 0,02 & 0,04 & 2,72 \\
MDEA & 80,3 & 16,66 & 5,52 & 3,06 \\
MDEA+MEA & 97,3 & 0,025 & 0,078 & 2,68 \\
MDEA+DEA & 97,2 & 0,05 & 1,06 & 2,76 \\
\hline
\end{tabular}


La energía requerida para invertir el calor de reacción es muy pequeña (15-30\%) cuando se la compara con la cantidad de calor requerido para aumentar la temperatura de la solución en el regenerador (Jenkins y Haws, 2001). Por lo tanto para optimizar la energía utilizada es necesario optimizar la tasa de circulación, maximizando la potencia de cada unidad de volumen circulado. Como se puede observar en la Tabla 1, el proceso que requiere menor caudal es el que utiliza DGA (80 gpm), mientras que el proceso con MEA es el que consume la mayor tasa de circulación (120 gpm).

Por otra parte, cuando se comparan las fuerzas relativas de las diferentes aminas, es necesario considerar la molaridad (mol/lt) para comprender cuál es la verdadera capacidad hacia los gases ácidos. Los valores de molaridad de las soluciones analizadas aumentan en el siguiente orden: MEA=DEA< MDEA< DGA. Adicionalmente, la fuerza básica relativa es otro aspecto a tener en cuenta para analizar la potencia de una solución de aminas. Cuanto mayor es la fuerza básica, mayor es la afinidad para remover gases ácidos. La contante de acidez pKa es la medida que normalmente se utiliza para medir la fuerza básica relativa de varias soluciones de tratamiento de gas. Los valores de pKa de las soluciones de aminas analizadas se relacionan de la siguiente manera: MDEA $<$ DEA $<$ MEA=DGA.

Estas últimas aclaraciones permiten entender porqué los mayores niveles de purificación se obtuvieron con la DGA y porqué para lograr estos niveles de purificación se necesita menores caudales y por lo tanto menor calor en el vaporizador. Similares resultados fueron obtenidos en la purificación de gases de combustión, incluso aunque la composición molar de $\mathrm{CO}_{2}$ en este caso fue bastante menor $(<3 \%)$ (Nuchitprasittichai y Cremaschi, 2011).

\section{CONCLUSIONES}

Los procesos convencionales de absorción-desorción son procesos que han sido investigados ampliamente para el gas natural y los gases de combustión. Esta tecnología madura permite hacer un uso adecuado de la misma para el caso particular del biogás, obteniendo resultados muy buenos en los niveles de metano alcanzados (cercanos al 97\%). Sin embargo, no dejan de ser muchas las variables que se deben analizar para diseñar una unidad óptima de purificación de biogás. En este trabajo se discutieron algunas de ellas para el proceso de absorción con aminas. Para ello, se simuló en ProMax ${ }^{\circledR}$ un proceso convencional de absorción-desorción para seis tipos de soluciones de aminas (MEA, DEA, DGA, MDEA) o mezclas de ellas (MDEA+MEA, MDEA+ DEA), mostrando altos niveles de purificación, siendo la DGA la que presenta las mayores ventajas al lograr mejores rendimientos $\left(97,3 \%\right.$ de $\left.\mathrm{CH}_{4}\right)$ con un menor consumo de energía $(5,25$ MMBTU/h).

\section{AGRADECIMIENTOS}

Este trabajo fue realizado gracias al apoyo económico de la Universidad Nacional del Litoral (UNL) y el Consejo Nacional de Investigaciones Científicas y Técnicas (CONICET).

\section{REFERENCIAS}

Abatzoglou, N., A review of biogas purification processes, Biofuels Bioprod. Bioref.: 3, 42-71 (2009).

Abu-Zahra M.R., L.H. Schneiders, J.P. Niederer, P.H. Feron y G.F. Versteeg, $\mathrm{CO}_{2}$ capture from power plants. Part I. A parametric study of the technical performance based on monoethanolamine, Internacional Journal of Greenhause Gas Control, 1, 37-46 (2007).

Henrich, R.A., Advances in Biogas to Fuel Conversion, Biocycle: 24 (4), 28-31 (1983).

Holmes, J.W., M.L. Spears y J.A. Bullin, Sweetening LPG's with Amines, Chemical Engineering Progress: 80 (5), 47 (1984).

Jenkins, J.L. y R. Haws, Understanding gas treating fundamentals, Petroleum Technology Quarterly: 6 (4), 61-71 (2001).

Kohl, A. y N. Nielsen, "Gas Purification”, 5ed., 40-277. Gulf Publishing Company, Houston, Texas (1997).

Lunsford, K.M. y J.A. Bullin, Optimization of Amine Sweetening Units, Actas del AIChE Spring National Meeting, 12-24, NY: American Institute of Chemical Engineers, USA (1996).

Nuchitprasittichai, A. y S. Cremaschi, Optimization of $\mathrm{CO}_{2}$ capture process with aqueous amines using response surface methodology, Computers and Chemical Engineering, 35, 1521-1531 (2011). 
Patterson, T., S. Esteves, R. Dinsdale y A. Guwy, An evaluation of the policy and techno-economic factors affecting the potential for biogas upgrading for transport fuel use in the UK, Energy Policy: 39, 1806-1816 (2011).

Polasek J.C. y J.A. Bullin, Selective Absorption Using Amines, (en línea) (1982). Bryan Research and Engineering, Inc. - Technical Papers, http://www.bre.com/support/technical-articles.aspx. Acceso: 13 de junio 2012.

Polasek J.C., J.A. Bullin y S.T. Donnelly, How to Reduce Costs in Amine Sweetening Units, Chemical Engineering Progress; 79 (3), 63 (1983).

Polasek, J.C. y J.A. Bullin, Selecting Amines for Sweetening Units, Energy Progress: 4 (3), 146-150 (1984).

Rasi, S., J. Läntelä, A. Veijaneny y J. Rintala, Landfill gas upgrading with countercurrent water wash, Waste Management: 28, 1528-1534 (2008).

Rubin, E.S., H. Mantripragada, A. Marks, P. Versteeg y J. Kitchin, The outlook for improved carbon capture technology, Progress in Energy and Combustion Science, In Press. http://www.sciencedirect.com/science/article/pii/S0360128512000184. Acceso: 11 de mayo 2012.

Ryckebosch, E., M. Drouillon y H. Vervaeren, Review: Techniques for transformation of biogas to biomethane, Biomass and Bioenergy: 35, 1633-1645 (2011).

Shethna, H.K. y G.P. Towler, Design of Mixed-Solvent Processes for Chemisorption with Ultrahigh Recovery, Ind. Eng. Chem. Res.: 36, 5307-5320 (1997).

Tippayawong, N. y P. Thanompongchart, Biogas quality upgrade by simultaneous removal of $\mathrm{CO}_{2}$ and $\mathrm{H}_{2} \mathrm{~S}$ in a packed column reactor, Energy: 35, 4531-4535 (2010).

Varnero, M.T., M. Carú, K. Galleguillos y P. Achondo, Tecnologías Disponibles para la Purificación de Biogás usado en la Generación Eléctrica, Inf. Tecnol.: 23 (2), 31-40 (2012). 